\title{
Proactive Channel Access in Dynamic Spectrum Networks
}

\author{
Work-in-Progress \\ Lei Yang, Lili Cao and Haitao Zheng \\ Department of Computer Science \\ University of California, Santa Barbara, CA 93106 U.S.A \\ Email: \{leiyang, lilicao, htzheng $\} @$ cs.ucsb.edu
}

\begin{abstract}
Open Spectrum systems allow fast deployment of wireless technologies by reusing under-utilized pre-allocated spectrum channels, all with minimal impact on existing primary users. However, existing proposals take a reactive sense-andavoid approach to impulsively reconfigure spectrum usage based solely on the latest observations. This can result in frequent disruptions to operations of both primary and secondary users. In this paper, we propose a proactive spectrum access approach where secondary users utilize past channel histories to make predictions on future spectrum availability, and intelligently schedule channel usage in advance. We propose two channel selection and switching techniques to minimize disruptions to primary users and maintain reliable communication at secondary users. Experiments show that the proactive approach effectively reduces the interferences to primary users by up to $30 \%$, and significantly decreases throughput jitters at secondary users.
\end{abstract}

\section{INTRODUCTION}

Conventional spectrum management policies use static spectrum assignment to prevent interference. Over time, this has led to the well-known artificial spectrum scarcity. Recent surveys have shown that licensed spectrum are overly-allocated and yet critically under-utilized, often as low as 5-10\% [11]. To overcome such artificial scarcity, the most promising solution is Open Spectrum systems [2], [11], where devices skip the licensing process and instead use next generation "Cognitive Radios" (CRs) [9], [14], becoming secondary users that opportunistically access spectrum currently unused by legacy or primary users.

Initial proposals for Open Spectrum systems take a reactive approach [12], [16], [17]. Secondary users reconfigure spectrum usages only after detecting changes in spectrum availability following some action by a primary user. Devices monitor spectrum channels through individual or collaborative sensing [3], [5], [7], [8], [10], [13]. When detecting a change in spectrum, e.g. a primary user appears, secondary users pause existing transmissions, relinquish the band and seek other opportunities to resume communications [19]. Reconfiguration is impulsive and is based solely on the latest observations.

Such passive "sense and react" approach results in frequent disruptions to communications of both primary and secondary users. Specifically, periodic sensing and adaptation means there is an unavoidable window of possible interference for primary users. As a result, primary users can experience shortterm interference to transmissions before being detected by neighboring secondary users. Similarly, secondary users suffer from unexpected interruptions to communications, making it extremely difficult to satisfy application requirements. They have no expectations of future spectrum availability to help coordinate spectrum access or schedule transmissions. Delay due to improper channel searching, sensing and switching leads to undesired gaps in transmission.

In this paper, we propose a proactive spectrum access approach where secondary users proactively predict future spectrum availability and intelligently schedule channel access in advance. By adding limited "intelligence," secondary users can take advantage of inherent patterns of primary users' spectrum usage, and make predictions about future changes in spectrum availability. They use these predictions, along with current observations, to determine spectrum usage to avoid disrupting primary users and maintain reliable communication.

The proposed approach includes two modules. First, to minimize disruption to primary users, secondary users proactively switch channel before any primary user appears. Second, to quickly resume communication, secondary users intelligently select another available (and reliable) channel. This paper makes the following contributions:

(1) Proactive spectrum access framework. We propose a framework for proactive spectrum access and provide detailed prediction methods assuming exponential and periodic traffic models. We also propose different prediction and schedule schemes using different sensing capabilities.

(2) Experiments to compare reactive and proactive approaches. We compare the two approaches by evaluating the disruption to primary users and the channel utilization at secondary users. The proactive approach leads to $30 \%$ reduction of disruptions compared to the reactive approach. The improvement depends heavily on the accuracy of spectrum availability prediction.

\section{BACKGROUND AND RELATED WORK}

In this section, we provide background on dynamic spectrum access and related work.

Dynamic Spectrum Availability Secondary device's spectrum availability depends on the activity of nearby primary devices. We start from describing a set of models in literature on primary user's activity. 
The mostly used model is the alternative exponential ONOFF model as studies have shown that it approximates the spectrum usage pattern at public safety bands [18]. Each channel alternates between two modes: ON (the channel is occupied by a primary user) and OFF (the channel is idle). The durations of the $\mathrm{ON}$ and the OFF period are independently exponentially distributed. For a channel $i$, the duration of $\mathrm{ON}$ period $y_{i}$ follows an exponential distribution with mean $\frac{1}{\lambda_{Y_{i}}}$ :

$$
f\left(y_{i}\right)=\left\{\begin{aligned}
\lambda_{Y_{i}} e^{-\lambda_{Y_{i}} y_{i}} & : \quad y_{i} \geq 0 \\
0 & : \quad y_{i}<0
\end{aligned}\right.
$$

Similarly, for each channel $i$, its length of OFF period $X_{i}$ follows an exponential distribution with mean $\frac{1}{\lambda_{X_{i}}}$. The second model is the periodic ON-OFF model where each channel displays a fixed pattern of busy and idle period. In this model, after a long-term observation, secondary users can make accurate predictions of future spectrum availability.

These models represent two extreme cases in terms of prediction capability. The alternative exponential model is highly random because of the "memoryless" nature of exponential distributions; while the periodic model can be accurately predicted given adequate observation time. In this paper, we use the exponential distribution model as an initial step. We plan to investigate the proposed approach using real spectrum measurement data in a future study.

Spectrum Sensing With dynamic spectrum availability, secondary users must monitor spectrum constantly and switch among channels to avoid disrupting primary users. Their behavior depends on the sensing capability. In the simplest case, each user uses one radio for both sensing and communication. If the radio can only sense one channel any time, each secondary user must use a sequential sense-transmitsense approach. Shown in Figure 1, each user first senses a channel and if it is idle, transmits for a short period and then sense again. On the other hand, with an external spectrum sensor, each user can monitor each channel continuously while communicating.

Reactive Spectrum Access Figure 1 illustrates the reactive channel access model. Any secondary user communicates via one channel until detecting any primary user. Because of the inherent delay in detection, secondary users could disrupt the operation at nearby primary users.

\section{A. Related Work on Proactive Spectrum Access}

There have been several prior works on dynamic spectrum access and sensing. The most relevant ones are [10], [15]. In [15] the authors proposed a proactive access scheme based on the characteristics of TV-broadcast and explored the feasibility of proactive access method. Our work extends this work to a general primary user traffic model, i.e., the exponential ONOFF model. Moreover, [15] mainly focuses on throughput maximization, while our work focuses on minimizing disturbance to primary users and providing fast recovery.

The work of [10] proposed an adaptive sensing scheme to detect unused channels, and order the channel search to

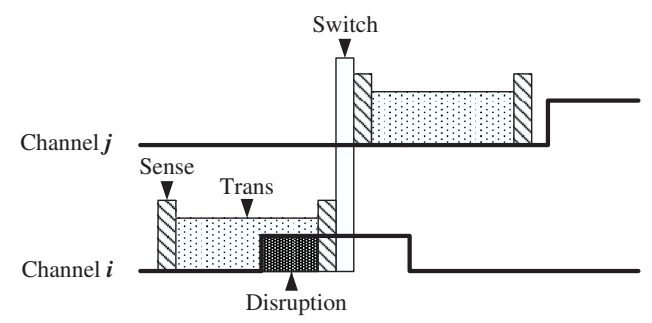

Fig. 1. An example of secondary user's spectrum access. The user first senses channel $i$ which is idle. After a transmission session, it senses again and detects a primary user. The user then switches to channel $j$ and repeats the process. For each channel, a bold line illustrates the dynamic spectrum usage of primary users.

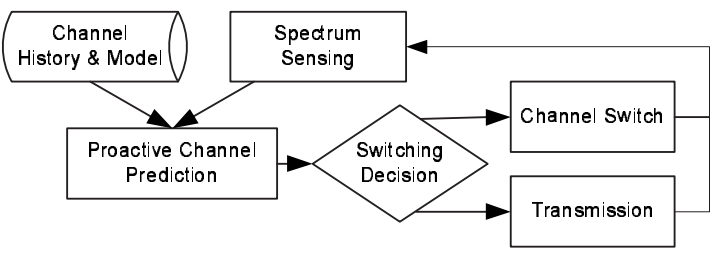

Fig. 2. Architecture of the proposed proactive spectrum access.

minimize reconnection delay. Our proactive channel selection technique uses a channel ordering approach similar to that of [10]. However, the main difference is that our approach predicts future spectrum availability and schedules channel switch in advance rather than reactively switch after detecting any primary user.

\section{Proactive Spectrum Access}

Under reactive spectrum access model, secondary users switch channels only after detecting a primary user, causing unavoidable interferences. Without expectations of future spectrum availability, secondary users can not make intelligent decisions on spectrum access. In this section, we show that by proactively predicting future spectrum availability, secondary users can intelligently switch channels before primary users' re-appearance. By scheduling spectrum usage, they can maintain reliable high-throughput communication while minimizing disruptions to primary users. Figure 2 illustrates the proposed architecture, including two core modules:

- Proactive Channel Prediction - Secondary users utilize past channel observations to estimate future spectrum availability.

- Intelligent Channel Switching - Utilizing prediction results, secondary users decide when to exit from a channel and which channel to switch to.

We make the following assumptions. First, secondary users use a separate control channel to coordinate with their communicating peers to synchronize channel switches. Second, while secondary users can leverage knowledge on peer contention to select channels, our approach focuses on selecting a channel with the least probability of encountering any primary users. We can combined the proposed approach with any distributed coordination approach [1], [4] to minimize contention among multiple secondary users. 


\section{A. Proactive Channel Prediction}

The first challenge we face is how to use past channel observations to estimate future spectrum availability. Specifically, we are interested in estimating the probability that a channel $i$ will be idle in the next time slot, referred to as $P_{i}$. We assume that each secondary user can acquire statistical property of spectrum usage at nearby primary users. These can be done offline through static traffic analysis, and made available to secondary users through online databases. Given primary user's statistical traffic model and parameters, we need to determine how each secondary user predicts $P_{i}$. Next, we outline the prediction algorithm for three traffic models.

Alternative Exponential Model Using renewal theory [6], [10], we can calculate $P_{i}$ as:

$P_{i}= \begin{cases}\frac{\lambda_{Y_{i}}}{\lambda_{X_{\dot{j}}+\lambda_{Y_{i}}}}+\frac{\lambda_{X_{i}}}{\lambda_{X_{\dot{j}}+\lambda_{Y_{i}}}} e^{-\left(\lambda_{X_{i}}+\lambda_{Y_{i}}\right) \Delta t_{i}} & s_{i}=I D L E \\ \frac{\lambda_{Y_{i}}}{\lambda_{X_{i}}+\lambda_{Y_{i}}}+\frac{\lambda_{Y_{i}}}{\lambda_{X_{i}}+\lambda_{Y_{i}}} e^{-\left(\lambda_{X_{i}}+\lambda_{Y_{i}}\right) \Delta t_{i}} & s_{i}=B U S Y\end{cases}$

where $\Delta t_{i}$ is the time gap from the last history $s_{i}$ to the next time slot.

Periodic model With sufficient observation time, secondary users can always accurately predict the channel availability.

Alternative Periodic-Exponential model This model is an intermediate model between the previous two extreme cases. The duration of ON (or OFF) periods is fixed to $T$, and the duration of OFF (or ON) periods is exponential distributed with $\lambda$. We can derive $P_{i}$ as [20]:

$P_{i}= \begin{cases}\frac{1}{T} \int_{0}^{T} \sum_{n=0}^{\left\lfloor\frac{\Delta t_{i}-x}{T}\right\rfloor} \frac{\lambda^{n}\left(\Delta \hat{t}_{i}-x\right)^{n}}{n !} e^{-\lambda\left(\Delta \hat{t}_{i}-x\right)} d x, & \Delta t_{i}>T \\ \frac{1}{T} \int_{0}^{\Delta t_{i}} e^{-\lambda\left(\Delta t_{i}-x\right)} d x, & \Delta t_{i}<T\end{cases}$

where $\hat{\Delta t_{i}}=\Delta t_{i}-n T$.

While our prediction mechanisms are similar to that of [10], we use the prediction results differently. While [10] uses $P_{i}$ to compute an order to search available channels, we use these predictions to switch channel before "bumping" into any primary users, and continuously update $P_{i}$ in each time slot.

\section{B. Intelligent Channel Switching}

Utilizing observations and predictions, secondary users can schedule channel usage to avoid disrupting primary users and maintain reliable communication. Figure 3 compares the behavior of reactive and proactive spectrum access. In reactive access, secondary users inevitably "bump" into primary users; while in proactive access, secondary users can avoid primary users by switching channel prior to primary user's appearance.

However, the effectiveness of proactive access depends heavily on being able to predict spectrum accurately. When predictions are imperfect, secondary users can make "dumb" switches. In Figure 3 we show two examples of dumb switching. In type I, a secondary user falsely interprets channel $j$ over $i$ and switches to an occupied channel, and thereby suffers from unnecessary interruptions to its communication. In type II, a user switches to a channel with shorter remaining
TABLE I

SUMMARY OF DIFFERENT SWITCHING BEHAVIORS

\begin{tabular}{|c|c|}
\hline Behavior & Description \\
\hline Reactive Switching & switch channel after detecting primary users \\
\hline $\begin{array}{ll}\text { Proactive } & \text { Smart } \\
\text { Switching }\end{array}$ & $\begin{array}{l}\text { switch to a channel with longer remaining idle } \\
\text { time than the current channel. }\end{array}$ \\
\hline $\begin{array}{l}\text { Proactive Dumb } \\
\text { Switching I }\end{array}$ & switch to a busy channel \\
\hline $\begin{array}{l}\text { Proactive Dumb } \\
\text { Switching II }\end{array}$ & $\begin{array}{l}\text { switch to a channel with shorter remaining idle } \\
\text { time than the current channel. }\end{array}$ \\
\hline
\end{tabular}

idle period than the current channel, which could reduce its communication period. We summarize different switching behaviors in Table I.

Our goal is to increase the use of smart switching and avoid dumb switching. The key factor that differentiates smart and dumb switching is the accurate prediction of the remaining idle period on each channel. If the remaining idle period in the current channel $c$ is shorter than that in another channel $i$, then switching from channel $c$ to $i$ is smart. Assuming the traffic of primary users follows alternative exponential model, we propose two criteria to plan channel usage:

Proactive Planning I A user switches to a channel $i$ with the largest expected remaining idle period, i.e.

$$
i=\arg \max _{j} \frac{P_{j}}{\lambda_{X_{j}}}
$$

Proactive Planning II A user switches from channel $c$ to $i$ if with high probability ( $>0.5$ ) that the length of the remaining idle period of $i$ is larger than that of $c$, i.e.:

$i=\arg \max _{j} \operatorname{Prob}\left(T_{j}>T_{c}\right)=\arg \max _{j}\left\{P_{j}-\frac{\lambda_{X_{j}}}{\lambda_{X_{j}}+\lambda_{X_{c}}} P_{j} P_{c}\right\}$.

\section{Simulation Results}

We use matlab-based simulations to evaluate both reactive and proactive spectrum access schemes under different network settings. Table II summaries different reactive and proactive schemes. Table III summaries the simulation parameters. To evaluate the performance of both secondary and primary users, we examine the average primary users' disruption rate (the number of disruptions per second) and the average channel utilization by secondary users. We have also evaluated the performance under various network settings, and verified these results using a wireless testbed. Results from these experiments are omitted due to space limit, but can be found in [20].

\section{A. Reactive vs. Proactive Approaches}

Alternative Exponential traffic model Figure 4 illustrates the CDF and average channel utilization and disruption rate of both proactive and reactive approaches. We see that the proactive approach can improve channel utilization by $5 \%$, but reduce the disruption rate by up to $30 \%$. The proposed prediction based smart switch can further reduce up to $12 \%$ of disruption rate over that of (RE_P_HIS) [10]. 


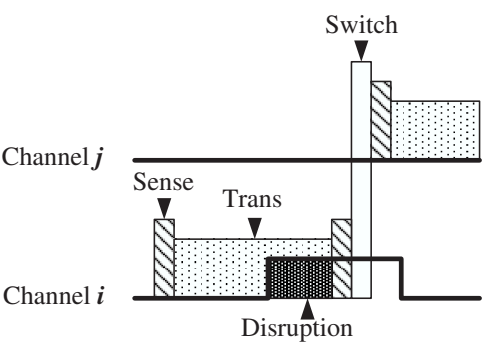

Reactive Switching

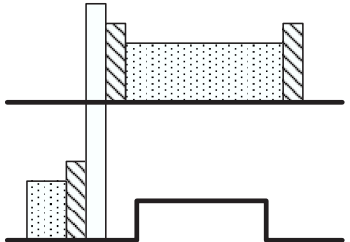

Proactive Smart Switching

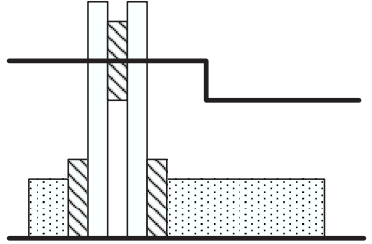

Proactive Dumb Switching I

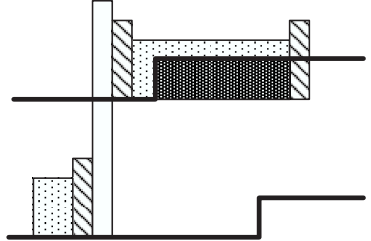

Proactive Dumb Switching II

Fig. 3. The four types of channel switching decisions: Reactive, Proactive Smart, Proactive Dumb I and Proactive Dumb II.
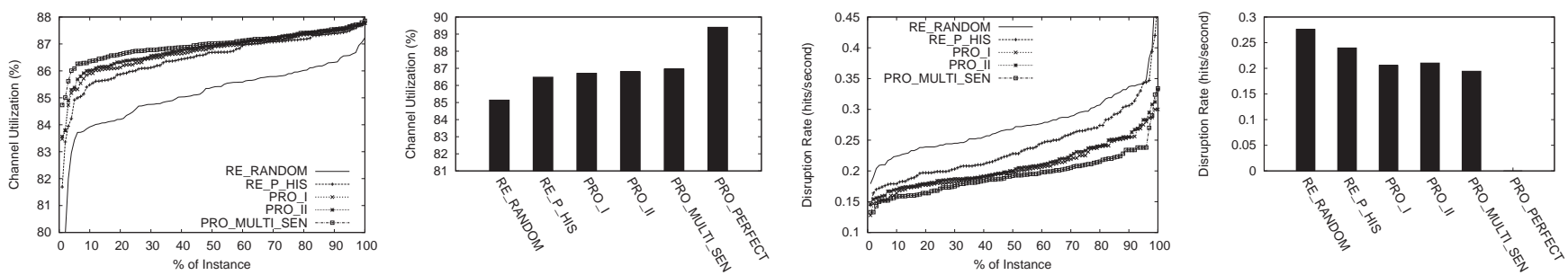

Fig. 4. (Left two) Secondary users' channel utilization in CDF and average, (Right two) Primary user's disruption rate in CDF and average; both assuming $\mu_{\min }=0.5, \mu_{\max }=5.0$.

TABLE II

SPECTRUM ACCESS SCHEMES

\begin{tabular}{|c|l|}
\hline Method & Description \\
\hline RE_RANDOM & Reactive switching; random channel selection. \\
\hline RE_P_HIS & $\begin{array}{l}\text { Reactive switching [10]; use }(1) \text { to derive } P_{i}, \\
\text { and choose the channel with the highest } P_{i} .\end{array}$ \\
\hline PRO_I & $\begin{array}{l}\text { Proactive switching; use }(1) \text { to derive } P_{i} ; \text { use }(3) \\
\text { to choose the channel with the longest expected } \\
\text { remaining idle period } E\left(T_{i}\right) .\end{array}$ \\
\hline PRO_II & $\begin{array}{l}\text { Proactive switching; use }(1) \text { to derive } P_{i} \text {; use (4) } \\
\text { to choose the channel with the largest probability } \\
\text { of having longer remaining idle period than that } \\
\text { of the current channel. }\end{array}$ \\
\hline PRO_MULTI_SEN & $\begin{array}{l}\text { Proactive switching with multi-channel sensing } \\
\text { ability and perfect prediction of } P_{i} ; \text { use (3) to } \\
\text { choose the channel with the longest expectation } \\
\text { of remaining idle period } T_{i} .\end{array}$ \\
\hline PRO_PERFECT & $\begin{array}{l}\text { Proactive switching with perfect knowledge of } \\
\text { the current channel status and the remaining } \\
\text { idle period; switch to a channel with longest } \\
\text { remaining idle time; the upper bound of system } \\
\text { performance. }\end{array}$ \\
\hline
\end{tabular}

TABLE III

Simulation PARAMETERS

\begin{tabular}{|c|c|}
\hline Parameter & Value \\
\hline Sensing Period $t_{s}$ & $20 \mathrm{~ms}$ \\
\hline Transmission Duration $T_{s}$ & $180 \mathrm{~ms}$ \\
\hline Switching Delay $D_{s}$ & $10 \mathrm{~ms}$ \\
\hline Number of Channels & 10 \\
\hline Primary user traffic models & $\begin{array}{c}\left(1 / \lambda_{X_{i}} \text { and } 1 / \lambda_{Y_{i}}\right) \text { uniformly } \\
\text { distributed in }\left[\mu_{\min }, \mu_{\max }\right]\end{array}$ \\
\hline Simulation Time & $10000 \mathrm{~s}$ \\
\hline
\end{tabular}

Alternative Periodic-Exponential model Figure 5 shows the system performance when the primary user on each channel follows the Fixed OFF and Exponential ON time
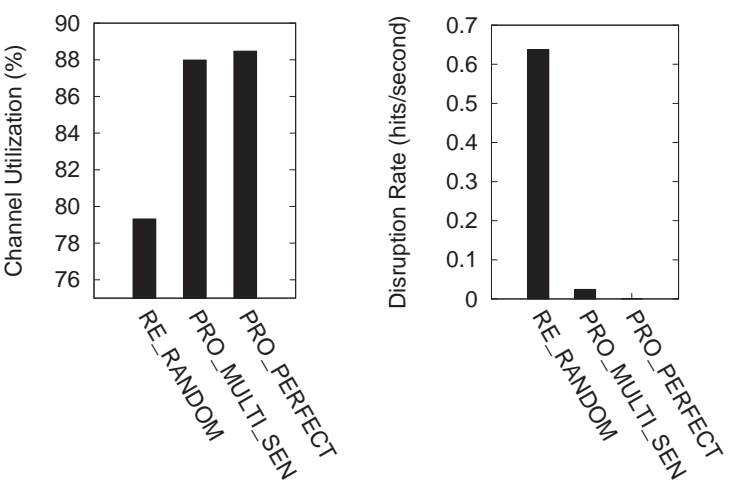

Fig. 5. Channel utilization and disruption rate of Fixed OFF-Exponential ON model.

traffic model. In this case, with multi-channel sensing ability, secondary users can obtain perfect information of past and current channel status, and make accurate prediction of the future channel status. From Figure 5, we see that the performance of proactive approach is almost perfect.

\section{B. Smart Switching}

The proposed proactive schemes (PRO_I and PRO_II) are designed to increase the number of smart switching. In Table IV, we examine the numbers of smart switching in different proactive schemes over $10000 \mathrm{~s}$. We see that the amount of smart switch is not large. This is because the imperfect prediction over on exponential ON-OFF traffic. However, with multichannel sensing, the number of smart switching improves to $30 \%$ due to improved estimation of $P_{i}$. However, because of the imperfect prediction of the remaining idle period, the number of dumb switching type II also increases significantly. 
TABLE IV

The Percentage of Proactive Switching

\begin{tabular}{|c|c|c|c|c|}
\hline Method & Switch No. & Smart & Dumb I & Dumb II \\
\hline PRO_I & 10690 & $3.7 \%$ & $5.2 \%$ & $1.5 \%$ \\
\hline PRO_II & 10258 & $6.1 \%$ & $2.9 \%$ & $2.4 \%$ \\
\hline PRO_MULTI_CHAN & 10269 & $30.0 \%$ & $0 \%$ & $21.8 \%$ \\
\hline PRO_PERFECT & 5131 & $100 \%$ & 0 & 0 \\
\hline
\end{tabular}
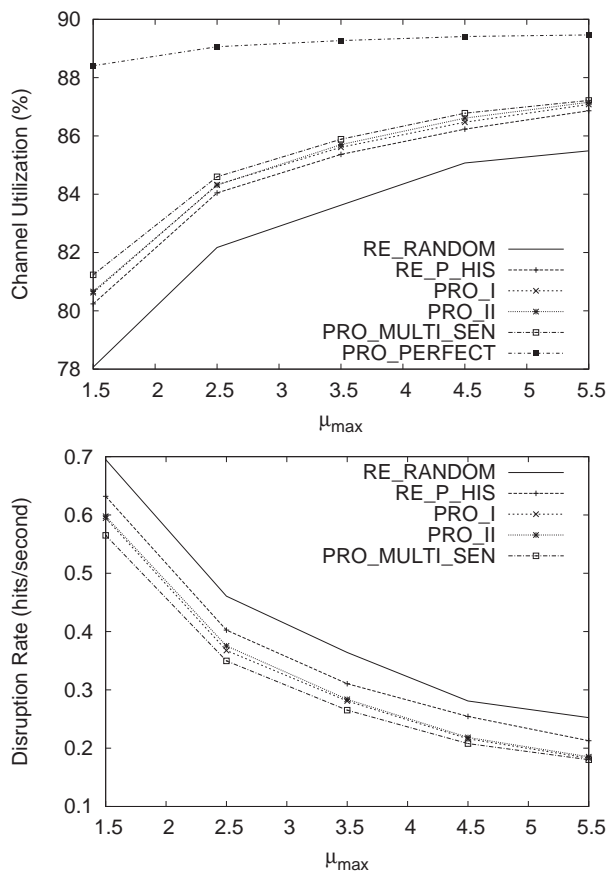

Fig. 6. Secondary user's channel utilization (top) and primary user's disruption rate (bottom) for different $\mu_{\max }$ ranging from 1.5 to 5.5.

\section{Impact of Primary User Traffic}

Using the Alternative Exponential ON-OFF model, we generate $1 / \lambda_{i}$ by using uniform distribution from $\left[\mu_{\min }, \mu_{\max }\right]$, fixing $\mu_{\min }$ to 0.5 , and varying $\mu_{\max }$ from 1.5 to 5.5 . Figure 6 shows the channel utilization and disruption rate for different $\mu_{\max }$. As $\mu_{\max }$ increases, primary user's activity reduces, thereby the channel utilization increases while the disruption rate drops. Similarly, proactive approaches achieve noticeable improvement over the reactive approach.

\section{CONCLUSION AND Future WORK}

We propose a proactive spectrum access to exploit underutilized licensed spectrum. While convention reactive solutions lead to disruptions to primary users because secondary users can not foresee future spectrum availability, we propose to intelligently schedule spectrum usage using prediction of future spectrum availability. Using past observations and knowledge of primary user's traffic statistics, secondary users can predict near future spectrum availability to switch channel prior to any appearance of primary users. These smart decisions help to avoid disrupting primary users and maintain reliable communication. Experimental results confirm that proactive approaches can significantly reduce disruptions to primary users.
We note that however, when primary user's traffic display large randomness, proactive approaches suffer from imperfect predictions, and make unnecessary "dumb" channel switches. Thereby it is necessary to first build sophisticated prediction mechanisms, possibility using insights from offline traffic analysis. We are currently researching on extending the proactive approach to other network scenarios where primary user's traffic and spectrum usages have predictable patterns.

\section{ACKNOWLEDGEMENT}

The authors would like to thank Dr. Eric Horvitz for his insightful suggestions for this paper.

\section{REFERENCES}

[1] Acharya, P. A. K., Singh, S., And Zheng, H. Reliable open spectrum communications through proactive spectrum access. In Proc. of TAPAS (August 2006).

[2] Berger, R. J. Open spectrum: a path to ubiquitous connectivity. ACM Queue 1, 3 (May 2003).

[3] CABric, D., Mishra, S. M., And Brodersen, R. W. Implementation issues in spectrum sensing for cognitive radios. In Proceedings of Asilomar conference on signals, systems and computers (2004).

[4] CAO, L., AND ZHENG, H. Spectrum allocation in ad hoc networks via local bargaining. In Proc. of SECON (September 2005).

[5] Challapali, K., Mangold, S., And Zhong, Z. Spectrum Agile Radio: Detecting Spectrum Opportunities. In International Symposium on Advanced Radio Technologies (ISART) (March 2004).

[6] Cox, D. Renewal Theory. Spottiswoode Ballantyne and Co. Ltd, 1962.

[7] Ganesan, G., And Li, Y. G. Cooperative spectrum sensing in cognitive radio networks. In Proc. of IEEE DySPAN (November 2005).

[8] Ghasemi, A., And Sousa, E. S. Collaborative spectrum sensing for opportunistic access in fading environments. In Proc. of IEEE DySPAN (November 2005)

[9] HAYKIN, S. Cognitive radio: Brain-empowered wireless communications. IEEE Journal on Selected Areas in Communications 23, 2 (Feburary 2005), 201-220.

[10] Kim, H., And Shin, K. G. Adaptive MAC-layer Sensing of Spectrum Availability in Cognitive Radio Networks. Technical Report, University of Michigan, CSE-TR-518-06.

[11] MCHenry, M. Spectrum white space measurements. New America Foundation Broadband Forum (June 2003).

[12] Mishra, S., Sahai, A., And Brodersen, R. Cooperative sensing among cognitive radios. In IEEE International Conference on Communications(ICC 2006).

[13] Mishra, S., Sahai, A., And Brodersen, R. Cooperative sensing among cognitive radios. In Proc. of IEEE ICC (May 2006).

[14] Mitola III, J. Wireless architectures for the 21st century. http: //ourworld.compuserve.com/homepages/jmitola.

[15] P. A. Kumar Acharya, S. S., And Zheng, H. Reliable Open Spectrum Communications Through Proactive Spectrum Access. In TAPAS 2006.

[16] Sahai, A., Hoven, N., And Tandra, R. Some fundamental limits on cognitive radio. In Forty-second Allerton Conference on Communication, Control and Computing (Monticello, IL, October 2004).

[17] SAhai, A., TANDRA, R., AND Hoven, N. Opportunistic spectrum use for sensor networks: the need for local cooperation. In Proc. of Information Processing in Sensor Networks(IPSN 2006).

[18] VujičCić, B., Cackov, N., VujičCić, S., And Trajković, L. Modeling and characterization of traffic in public safety wireless networks. In Proc. of SPECTS (2005).

[19] Willkomm, D., Gross, J., And Wolisz, A. Reliable link maintenance in cognitive radio systems. In Proc. of IEEE DySPAN (November 2005).

[20] YAng, L., CAO, L., And Zheng, H. Proactive opportunistic spectrum access. Technical report, University of California, Santa Barbara, 2007. 